\title{
FAST Observations of Upward Accelerated Electron Beams and the Downward Field-Aligned Current Region
}

\author{
R. C. Elphic ${ }^{1}$, J. Bonnell ${ }^{1,3}$, R. J. Strangeway ${ }^{2}$, C. W. Carlson ${ }^{3}$, M. Temerin', \\ J. P. McFadden ${ }^{3}$, R. E. Ergun ${ }^{3}$, and W. Peria ${ }^{4}$
}

\begin{abstract}
Auroral processes result from the exchange of energy and momentum between the magnetosphere and ionosphere, and the current systems arising from this exchange. Field-aligned potential drops in the upward field-aligned current (FAC) region of the auroral zone maintain a required current density in the face of opposing mirror forces. But field-aligned potential drops occur in downward current regions as well, and result in upgoing accelerated electron beams. We investigate the occurrence of upward accelerated electrons with altitude, magnetic local time and season using FAST. By choosing orbits having perigee over the equator, northern-southern hemisphere differences in altitude coverage are largely removed. For an interval near solstice, no electron beams were observed over the summer auroral zone at FAST altitudes, while over the winter hemisphere, $71 \%$ of the crossings had upgoing beams, a clear seasonal effect. During the equinox interval the distribution between hemispheres was much more balanced, but the overall occurrence was less $(\sim 46 \%)$ than during winter solstice. There is a clear tendency for upgoing accelerated electron beam occurrence at FAST altitudes to maximize when dipole tilt places the field line footpoint deep in the nightside ionosphere, where background ionospheric density is lowest. Thus the accelerating potential structure dips down to lower altitudes in this case.
\end{abstract}

\section{INTRODUCTION}

The auroral zone is the most dramatic and energetic manifestation of the inter-regional processes coupling the hot, tenuous magnetospheric plasma and the cold, dense, collisional ionospheric plasma. Auroral processes are thus intimately linked to the driving mechanisms of magnetospheric current systems. To maintain current continuity, large potential drops develop along auroral zone field lines. Field-aligned potential drops develop in regions of upward FACs to accelerate the tenuous plasma sheet electrons into the ionosphere, in effect widening the loss cone to provide sufficient current flow in the circuit [Knight, 1973]. In general, no such potential was thought to be necessary for

\footnotetext{
${ }^{1}$ Los Alamos National Laboratory, Los Alamos, New Mexico

${ }^{2}$ Institute of Geophysics and Planetary Physics, University of California, Los Angeles, California

${ }^{3}$ Space Sciences Laboratory, University of California, Berkeley, California

${ }^{4}$ University of Washington, Seattle, Washington
}

the downward FACs, because the density of ionospheric electrons would be sufficient to supply all the necessary current.

But sporadic evidence for field-aligned potential drops and upward accelerated electron beams in downward FAC regions has been observed over the years [Shelley et al., 1976; Sharp et al., 1980; Klumpar and Heikkila, 1982; Johnstone and Winningham, 1982; Burch et al., 1983; Gorney et al., 1985; Hultqvist et al., 1988; Marklund et al., 1994; Boehm et al., 1995]. Recent observations by the Fast Auroral SnapshoT Explorer (FAST) mission have demonstrated that such potential drops are a very common feature in the auroral zone during winter [Carlson et al., 1998; McFadden et al., 1998; Ergun et al., 1998]. Regions of downward current thus appear to sometimes re- 
quire a parallel potential structure to maintain current flow, evidently when the ionospheric plasma distribution lacks sufficient charge carriers to do so otherwise.

Preliminary analysis suggests that the characteristic scale size of downward current regions, and their associated potential structures, is smaller than that of the typical inverted-V/upward current region. Current densities in the downward current regions are thus correspondingly larger. Early analysis of FAST upgoing electron beam observations indicated that they are found between 2000 and 4000 $\mathrm{km}$ altitude over the winter auroral zone on about $78 \%$ of the passes [Carlson et al., 1998]. The occurrence probability drops quickly below $2500 \mathrm{~km}$, and the overall beam occurrence varies with season. (See also Peria et al., this volume). It has been demonstrated that the observed electron beams have number fluxes that agree closely with the current density inferred from magnetometer measurements [Carlson et al., 1998; Elphic et al. 1998]. Thus these beams can carry most of the field-aligned current density in a downward current region: hence their importance.

To place this study in the broader context of inter-regional processes (the exchange of energy and momentum between the magnetosphere and ionosphere) and current-driving mechanisms, we consider the following simple picture. In the spirit of Parker [1996; and this volume] and Iijima [this volume], the observed magnetic deflections at low altitudes can be thought of as manifestations of Maxwell stresses applied to the ionosphere, $\mathrm{B}_{\mathrm{dip}} \cdot \Delta \mathrm{B} / \mu_{\mathrm{o}}$ (see also Strangeway et al., this volume). These stresses are balanced in steady state in the ionosphere by friction due to ion-neutral collisions: $\nabla \cdot\left(\mathrm{B}_{\text {dip }} \cdot \Delta \mathrm{B} / \mu_{\mathrm{o}}\right)=\rho(\mathbf{v}-\mathbf{U}) v_{\text {in }}$, where $\mathrm{B}_{\text {dip }}$ is the ionospheric magnetic field, $\Delta \mathrm{B}$ is the perturbation field component, $\rho$ is the ionospheric plasma density, $\mathbf{v}$ is the ion drift velocity, $\mathbf{U}$ is the neutral drift velocity and $v_{\text {in }}$ is the ion-neutral collision frequency. The low-altitude Maxwell stresses should map to the motions of

plasma in the magnetosphere, as illustrated in Figure 1 for the premidnight auroral zone. The top panel shows the stressed, tilted magnetic field lines and their connection to the ionosphere; here the high latitude stresses are applied in the anti-sunward/eastward direction, while at lower latitudes the stress is sunward/westward. The bottom panel shows the magnetospheric plasma motions needed to maintain the magnetic stress against friction in the ionosphere, namely antisunward convection in the outer magnetosphere and sunward convection in the inner magnetosphere. Fieldaligned currents are present where there are shears in the magnetic field, in this case with downward FACs at the poleward and equatorward edges, and upward FACs in the middle. Each sense of FAC is associated with electrostatic potential structure necessary to supply the current density required by magnetic field shears that are imposed by motions.

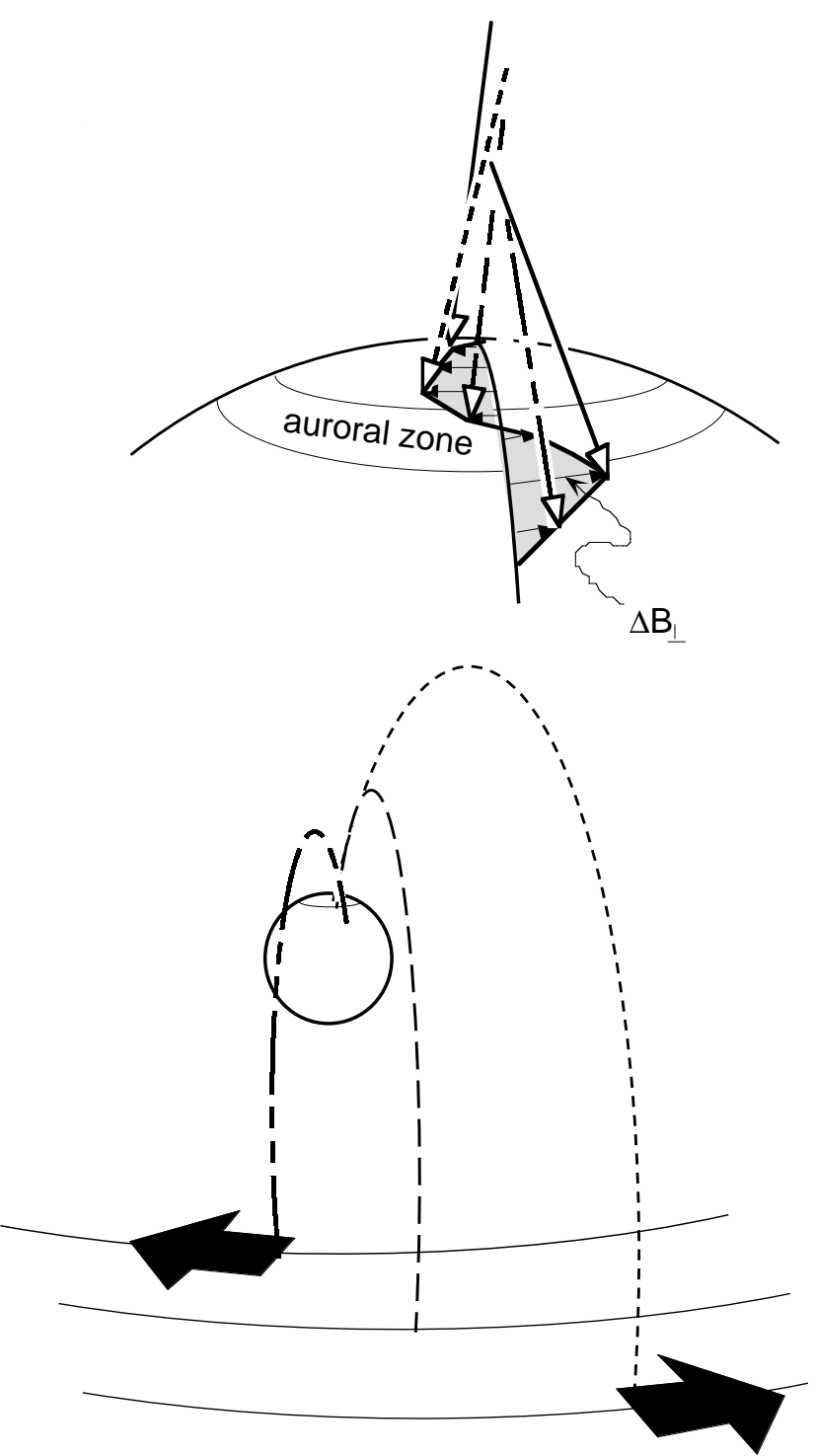

Figure 1. (top) Schematic diagram of sheared magnetic field lines applying Maxwell stress to the ionospheric plasma in the premidnight region. The horizontal perturbation field is shown by black arrows, which is opposite to the sense of the stress. (bottom) The magnetospheric motion of the flux tubes that is consistent with the stress applied to the ionosphere.

Using FAST observations, we have studied the altitude, invariant latitude (ILAT) distribution, seasonal dependence, and activity dependence of upward accelerated electron beams in the near-midnight magnetic local time (MLT) regions. We shall see that, for constant plasma sheet conditions and downward current density, a lowerdensity ionospheric profile will correspond to a loweraltitude accelerating potential, whereas for a higher-density ionospheric profile the potential structure will appear at higher altitudes. 


\section{APPROACH}

We make judicious use of the rapidly precessing FAST polar orbit to study beam dependence on altitude, MLT, and seasonal or dipole tilt effects. By picking out periods when perigee is at the equator, and apogee is in the midnight sector, we can confine our study to near midnight MLTs with similar altitude coverage in the north and south. Then by dividing these intervals into equinox and solstice subsets, we can study seasonal effects (which primarily affect the ionospheric density distributions, and hence possibly the altitudes at which the accelerating potentials form). For the equinox cases, we expect the ionospheric density distributions to be similar between the two hemispheres since the field line footpoints in the auroral zone have similar solar zenith angles. For the solstice cases one hemisphere will have auroral footpoints in the depleted, dark winter ionosphere, the other in the denser, sunlit summer ionosphere. We focus on two intervals in the FAST data meeting the foregoing conditions: the equinox interval 15 - 29 September, 1997, and the solstice interval 25 December, 1997 - 8 January, 1998. The FAST orbit provides roughly 11 northern and southern auroral passes per day, so each 15-day interval could potentially provide as many as 165 auroral passes through the near-midnight region for each hemisphere.

Plate 1 shows magnetic field and electron-related quantities that illustrate some of the features of the pre-midnight winter auroral zone. The top panel shows the east-west perturbation magnetic field, the second panel shows the field-aligned current density inferred from the slope of the field perturbation, the third panel shows the inferred height-integrated Pedersen conductivity $\Sigma_{\mathrm{P}}$ based on precipitating electron energy fluxes [Robinson et al., 1987], and the electron energy and pitch angle spectrograms are shown at the bottom. This pass illustrates that downward currents are often found at the edges of the inverted- $\mathrm{V}$ electron structures, and these downward current regions tend to be smaller and carry higher current densities than the inverted-V regions. Moreover, they are associated with minima in the inferred $\Sigma_{\mathrm{P}}$, not at gradients in $\Sigma_{\mathrm{P}}$ as would be expected if a constant electric field is being maintained in the ionosphere. The upward electron flux in these downward FAC regions is at times so high that the entire column of winter polar ionospheric plasma could be severely depleted within tens of seconds to minutes [Elphic et al., 1998]. The downward FACs are found on the poleward side of the antisunward/eastward Maxwell stress region $\left(\Delta \mathrm{B}_{\text {EAST }}<0\right)$, and on the equatorward side of the sunward/westward Maxwell stress region $\left(\Delta \mathrm{B}_{\mathrm{EAST}}>0\right)$. The downward current is carried here by upgoing electrons that have been

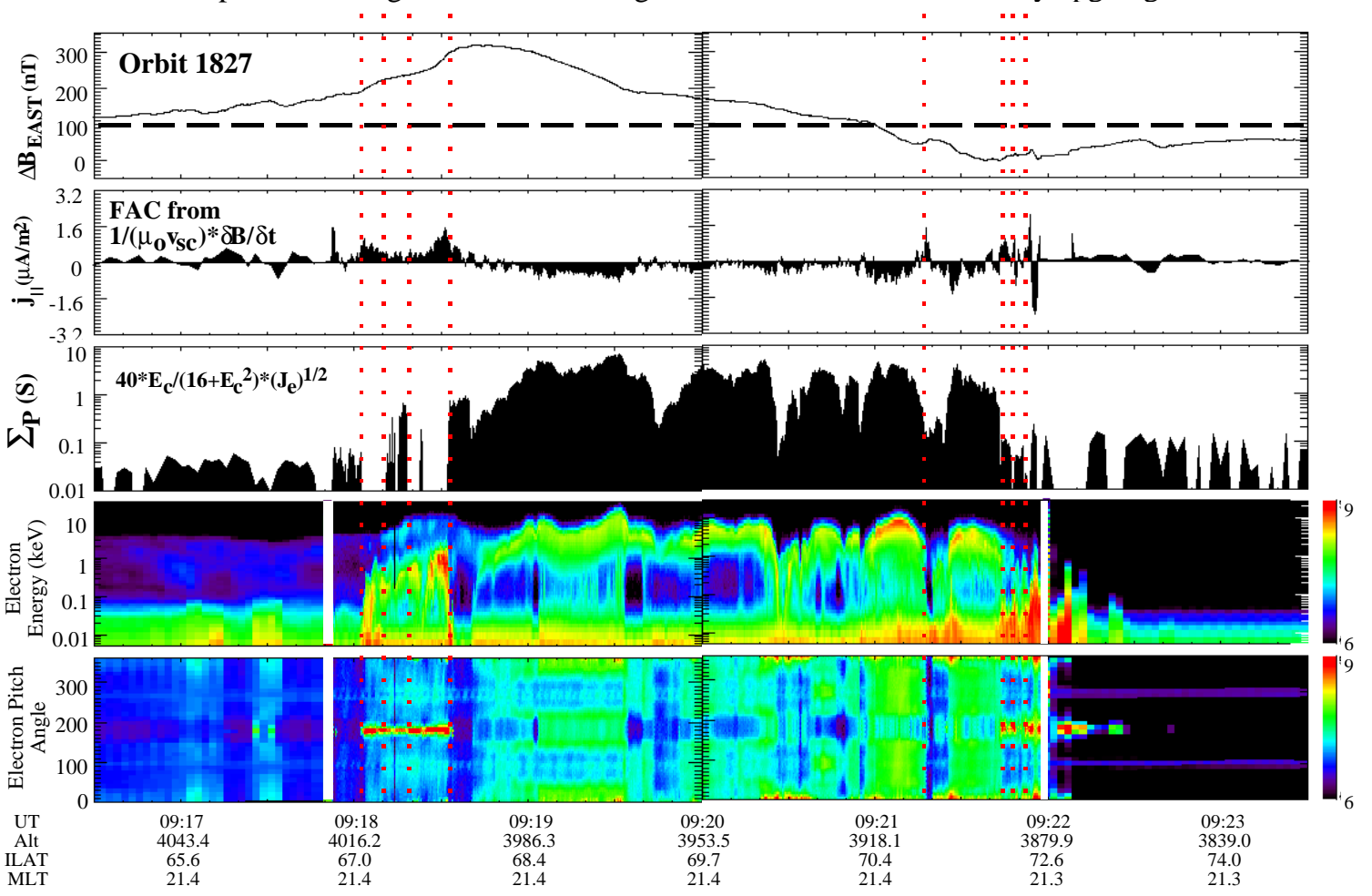

Plate 1. Data from a FAST auroral pass on February 6, 1997. The panels show the perturbation east-west magnetic field component, the inferred sheet current density (downward currents are positive), the inferred height-integrated Pedersen conductance based on electron precipitation, and electron energy and pitch angle spectrograms (color coded for $\log \mathrm{eV} \mathrm{cm}^{-2} \mathrm{~s}^{-1} \mathrm{sr}^{-1} \mathrm{eV}^{-1}$ ). The pass is at about $21.4 \mathrm{MLT}$, at an altitude of about $4000 \mathrm{~km}$. 
accelerated, in some cases achieving energies above $1 \mathrm{keV}$. For this survey, we identify an electron beam event/downward FAC region using upgoing electrons.

The events were selected using FAST summary data, which have a 5-sec temporal resolution. We identified the accelerated upgoing electron beams on the basis of both pitch angle (confined to a few degrees within the loss cone) and energy $(\mathrm{E}>20 \mathrm{eV})$, and energy flux greater than $10^{7}$ $\mathrm{eV} \mathrm{cm} \mathrm{cm}^{-2} \mathrm{sr}^{-1} \mathrm{eV}^{-1}$ (most beams have much higher energy fluxes, typically in the range of $10^{9} \mathrm{eV} \mathrm{cm}^{-2} \mathrm{~s}^{-1} \mathrm{sr}^{-1} \mathrm{eV}^{-1}$ ). The data were scanned and beam events were logged by orbit and time, and we also noted if the peak beam energy exceeded $1 \mathrm{keV}$. The corresponding altitude, magnetic local time (MLT), invariant latitude (ILAT), activity level (Kp) were also determined for each case.

\section{ALTITUDE AND ILAT DISTRIBUTIONS}

The solstice cases in our study are confined to within a few hours local time of midnight, though orbital coverage extends outside this range. There is a slight asymmetry in MLT sampling between the north and south cases during the equinox interval, with northern observations biased to earlier local times and southern to later local times. In altitude, the solstice cases can be found as low as about 1600 $\mathrm{km}$ with many cases below $2000 \mathrm{~km}$, whereas the equinox cases are mostly found above $2000 \mathrm{~km}$. No beams were found below about $1500 \mathrm{~km}$. These results are similar to those discussed by Carlson et al., [1998]; there is an apparent cutoff of occurrence at altitudes above roughly $3500 \mathrm{~km}$ is due to orbital coverage for the intervals used here (the orbit passes through altitudes above $3500 \mathrm{~km}$ mainly at invariant latitudes equatorward of the auroral zone). The northern and southern altitude - ILAT distributions for the equinox cases are very similar. Most beams are found within the range of $60^{\circ}-80^{\circ}$ ILAT, the normal range of auroral phenomena near midnight MLT.

Figure 2 shows the fraction of electron beam cases divided by the fraction of orbital samples in that bin with altitude for the two seasons (the error bars denote the uncertainty based on $\mathrm{N}^{1 / 2}$, where $\mathrm{N}$ is the number of cases in the bin). This ratio is proportional to the total number of beam events in a bin divided by the total number of orbital opportunities to observe a beam within that bin (in other words, fractional occurrence). For both the solstice and equinox cases, the beam occurrence drops to zero at low altitudes before the orbital coverage does, meaning that the incidence of upgoing electron beams really decreases below about $1600 \mathrm{~km}$. Above about 3300-km altitude, the orbital coverage for the selected time intervals does not permit an estimate of beam occurrence. For both seasons, the beam fractional occurrence increases above $1600-\mathrm{km}$ altitude and levels off at a roughly constant occurrence above about $2500-\mathrm{km}$ altitude. This may imply that the entire field- aligned accelerating potential structure tends to lie within the altitudes between 1600 and $2500 \mathrm{~km}$, with possibly a slightly higher altitude distribution for the equinox cases.
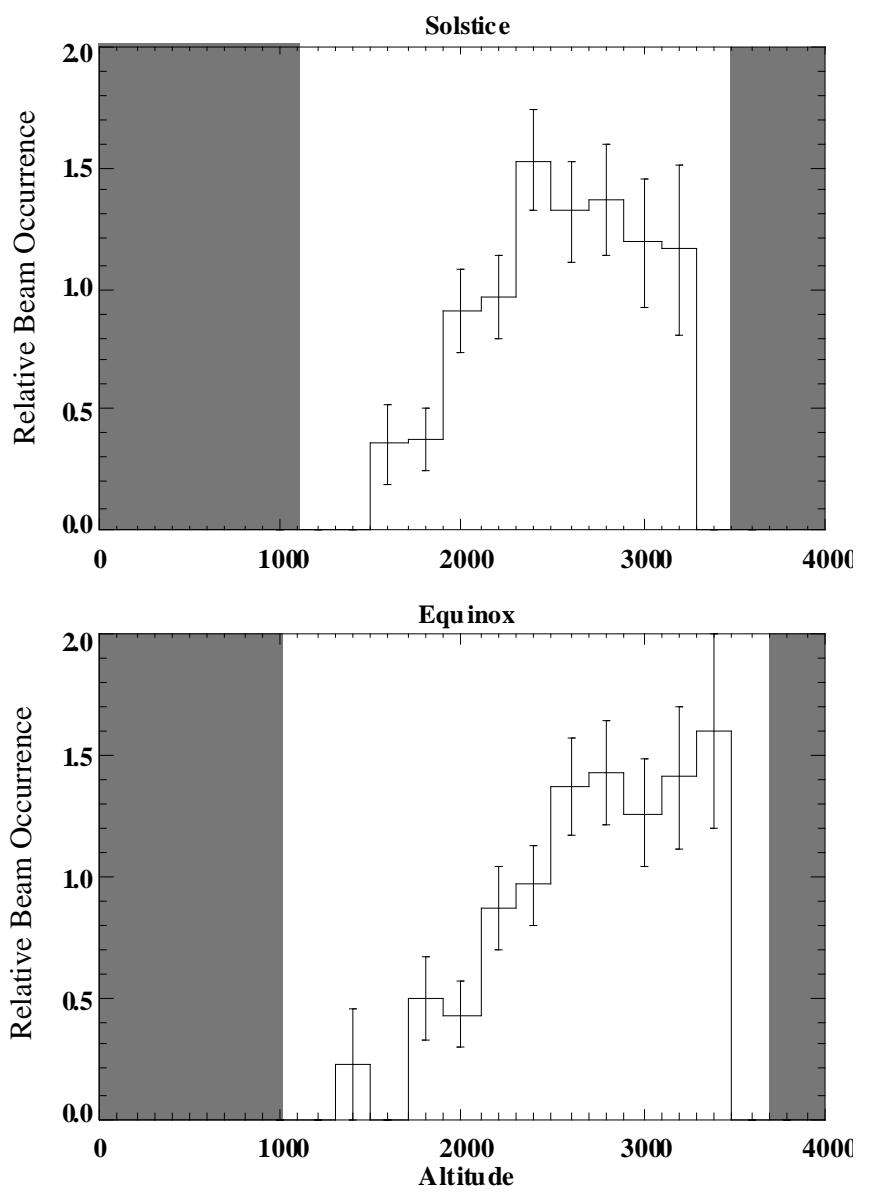

Figure 2. Histograms of number of upgoing beams divided by the number of orbits within each bin. Error bars denote the corresponding $\mathrm{N}^{1 / 2}$ uncertainty, where $\mathrm{N}$ is the number of events in a bin. Upgoing electron beam occurrence drops below about $2000 \mathrm{~km}$ altitude, and reaches a fairly steady occurrence above $2500 \mathrm{~km}$. Hatched regions denote no coverage for these intervals.

\section{SEASONAL AND ACTIVITY EFFECTS}

Table 1 presents a list by day of the number of FAST near-midnight auroral passes with upgoing accelerated electron beams and the number of passes without beams. For solstice, the northern winter passes with beams outnumbered the passes without beams ( $71 \%$ had beams), whereas southern summer passes yielded no beams at all. Note that the overall coverage is similar, 142 passes in the north and 133 in the south. FAST telemetry coverage during the equinox period was somewhat lower than during solstice, with data from a total of 117 northern passes and 126 southern passes returned to the ground (out of 165 possible). Of the data returned, accelerated upgoing electron beams were observed on $59 \%$ of the northern and 
$33 \%$ of the southern passes (the bottom line of Table 1). This difference in occurrence between the northern and southern equinox passes is puzzling because we chose the orbital configuration to remove any biases in altitude, MLT, or ILAT between the two hemispheres.

Table 1. Upgoing Accelerated Electron Beam Occurrence

\begin{tabular}{cccccc}
\hline \multicolumn{2}{c}{ Solstice (number of passes) } & \multicolumn{3}{c}{ Equinox (number of passes) } \\
& North & South & & North & South \\
Date & Yes/No & Yes/No & Date & Yes/No & Yes/No \\
\hline $12 / 25 / 1997$ & $7 / 3$ & $0 / 10$ & $9 / 15 / 1997$ & $5 / 3$ & $3 / 4$ \\
$12 / 26 / 1997$ & $5 / 4$ & $0 / 10$ & $9 / 16 / 1997$ & $7 / 3$ & $4 / 5$ \\
$12 / 27 / 1997$ & $3 / 7$ & $0 / 10$ & $9 / 17 / 1997$ & $4 / 4$ & $4 / 3$ \\
$12 / 28 / 1997$ & $6 / 4$ & $0 / 10$ & $9 / 18 / 1997$ & $5 / 2$ & $3 / 5$ \\
$12 / 29 / 1997$ & $5 / 5$ & $0 / 9$ & $9 / 19 / 1997$ & $1 / 5$ & $2 / 4$ \\
$12 / 30 / 1997$ & $5 / 2$ & $0 / 8$ & $9 / 20 / 1997$ & $7 / 1$ & $4 / 4$ \\
$12 / 31 / 1997$ & $6 / 4$ & $0 / 9$ & $9 / 21 / 1997$ & $4 / 5$ & $3 / 6$ \\
$1 / 1 / 1998$ & $4 / 2$ & $0 / 6$ & $9 / 22 / 1997$ & $4 / 5$ & $2 / 8$ \\
$1 / 2 / 1998$ & $10 / 1$ & $0 / 11$ & $9 / 23 / 1997$ & $3 / 3$ & $4 / 5$ \\
$1 / 3 / 1998$ & $7 / 3$ & $0 / 11$ & $9 / 24 / 1997$ & $4 / 4$ & $2 / 6$ \\
$1 / 4 / 1998$ & $7 / 2$ & $0 / 9$ & $9 / 25 / 1997$ & $5 / 2$ & $1 / 3$ \\
$1 / 5 / 1998$ & $9 / 1$ & $0 / 10$ & $9 / 26 / 1997$ & $5 / 1$ & $3 / 5$ \\
$1 / 6 / 1998$ & $8 / 1$ & $0 / 11$ & $9 / 27 / 1997$ & $5 / 3$ & $2 / 6$ \\
$1 / 7 / 1998$ & $9 / 1$ & $0 / 9$ & $9 / 28 / 1997$ & $6 / 4$ & $4 / 6$ \\
$1 / 8 / 1998$ & $10 / 1$ & $0 / 11$ & $9 / 29 / 1997$ & $4 / 3$ & $1 / 6$ \\
& & & & & \\
Total & $101 / 41$ & $0 / 133$ & Total & $69 / 48$ & $42 / 84$ \\
& $71 \%$ & $0 \%$ & & $59 \%$ & $33 \%$ \\
\hline
\end{tabular}

The apparent difference between equinox north and south occurrence is due to systematics in orbital coverage for FAST. Downlink of the data depends on availability of suitable ground stations, which occurs preferentially at about the same times every day. By the same token, outages due to lack of ground station availability also tend to occur systematically at other times every day. During the equinox season studied here, these outages just happen to occur at times far from the maximum dipole tilt for the north equinox cases, but are centered on the southern maximum tilt time. Thus the coverage bias tends to favor times when northern away tilt is maximized, and vice versa for the southern tilt conditions. We can take this bias into account by normalizing to the actual amount of time observations were possible at a given dipole tilt. After correcting for these biases, we see that the fractional occurrence shown in Figure 3 is essentially identical between the two hemispheres, and clearly demonstrates that dipole tilt (hence solar zenith angle) affects the observed beam occurrence for equinox conditions at FAST altitudes.

The upgoing electron beam energy is a rough indicator of the potential drop. We have tabulated the occurrence of electron beams with a peak energy below $1 \mathrm{keV}$, and those having peak energies above $1 \mathrm{keV}$. We have calculated the fraction of cases of each category as a function of $\mathrm{Kp}$, together with the distribution of all $\mathrm{Kp}$ values found during the solstice and equinox intervals. For both solstice and equinox, there is a dearth of high-energy beams for the quietest conditions, $\mathrm{Kp} \sim 0$. There is some suggestion that more energetic beams are found as $\mathrm{Kp}$ increases, but this tendency is far from clear. It is also of interest to determine if beam occurrence is higher during active times. We have compiled the fraction of beam cases in bins of $\mathrm{Kp}$, together with the overall fraction of $\mathrm{Kp}$ values for the solstice and equinox periods. The histograms (not shown) are very similar (and the uncertainties generally overlap), indicating that beam occurrence does not appear to increase significantly for higher Kp values.

\section{SUMMARY AND DISCUSSION}

We have studied the occurrence of upgoing accelerated electron beams by using precession of the FAST orbit at times when perigee (and apogee) is over the equator, providing comparable coverage in altitude, ILAT, MLT and dipole tilt over each hemisphere. We then selected intervals near northern winter solstice and equinox to clarify the role of ionospheric density in beam occurrence. We find that beams are found starting at altitudes near 1600 $\mathrm{km}$, and rise to a constant occurrence frequency around $2500 \mathrm{~km}$. This may imply that the accelerating potentials form in the neighborhood of $1600-2500 \mathrm{~km}$, and not higher.

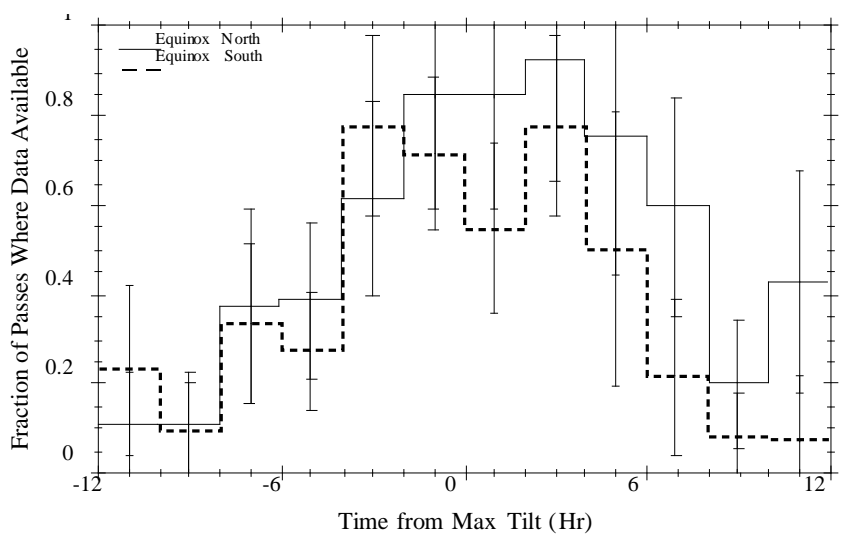

Figure 3. (top) Fractional occurrence of northern (southern) equinox passes with beams plotted as solid (dotted) lines versus the time of maximum away dipole tilt, 0440 UT for northern hemisphere cases, 1640 UT for southern hemisphere cases. The beam occurrence is peaked at the time of maximum away tilt for each corresponding hemisphere.

The overall occurrence of upgoing accelerated electron beams around midnight MLT is greatest near winter solstice ( $71 \%$ of the passes) and least near summer solstice $(0 \%)$. The equinox occurrence was lower, with $59 \%$ and $33 \%$ of passes exhibiting beams in the north and south, respectively. The lower occurrence and the apparent asymmetry appears to be related to both dipole tilt and orbital 
coverage. The highest occurrence of beams is found when
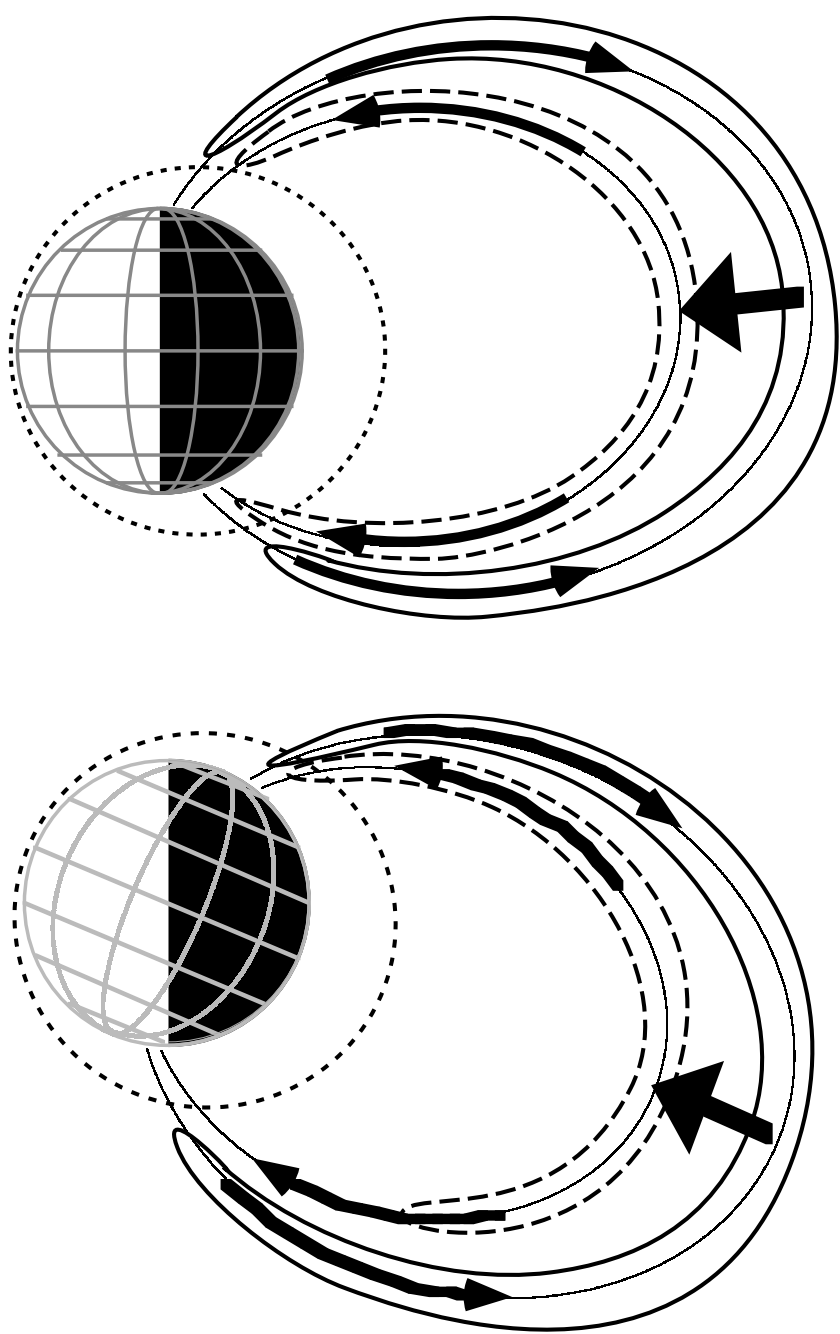

Figure 4. Schematic showing that the potential structure that accelerates the electrons sloshes up and down the field lines as the dipole rotates. (top) For the equinox case the potential structure associated with upgoing electron beams (dashed contour) drops down below the FAST orbit altitudes only in the hemisphere where the dipole rocks away from the sun. (bottom) For the solstice case, the potential structure only drops to FAST altitudes in the winter hemisphere, but stays there throughout the dipole rotation. The potential structures associated with inverted-V's may also drop to lower altitudes in the winter hemisphere. Current flow in both cases is shown by the solid arrows.

the magnetic pole is most tilted away from the sun, centered on 04:40 UT in the north and 16:40 UT in the south. This geometry means that the ionospheric footpoints have their largest solar zenith angles, are farthest from the terminator and hence have the lowest ionospheric densities. Moreover, these results imply that the altitude of the accelerating potential is lowest when the dipole tilt away from the sun is greatest. These accelerating potentials are thought to form closed banana-shaped surfaces, as shown in Figure 4. The figure shows that, for steady conditions, these surfaces would rise and fall in altitude as the dipole rocks during equinox.

Surprisingly, beam occurrence does not show a significant increase with geomagnetic activity. The occurrence of electron beams with peak energies greater than $1 \mathrm{keV}$ does not clearly increase with $\mathrm{Kp}$. These results imply that higher potentials are not found as activity (and the net field-aligned current) increases. This may indicate that during high $\mathrm{Kp}$ larger net field-aligned currents are carried by broader regions of modest current density, rather than smaller regions of more intense current density with higher associated potential drops.

We are left with the question of why downward currents in the winter premidnight auroral zone form thin sheets of high current density instead of spreading out into broader layers of lower current density. The answer may lie in the nature of how magnetospheric stresses are accomodated by the ionosphere. In the winter nightside ionosphere the background density is low, and enhancements to this density occur only where significant electron precipitation occurs (see Plate 1). The frictional force $\rho(\mathbf{v}-\mathbf{U}) v_{\text {in }}$ is thus higher in inverted-V regions, and lower outside these regions. An applied magnetospheric stress can cause the ionosphere to "slip" more easily in the low-density, low-friction regions away from the inverted-V precipitation. In other words, the low-density ionosphere can more easily reduce the applied stress, which results in a larger magnetic field shear between the high-density, high-friction inverted-V region and the lowdensity, low-friction region just outside of it. The enhanced magnetic shear is just another way of describing a high, localized downward field-aligned current density. In sunlit or higher-density ionospheric conditions, where inverted-V precipitation does not add much additional ionization to the background density, we would not necessarily expect to see such localized, high-current density downward FACs.

Acknowledgments. This work was supported by NASA order number S-57795-F. We are grateful to the FAST team for comments and suggestions. This paper is dedicated to the memory of Alan Johnstone.

\section{REFERENCES}

Boehm, M. H., J. Clemmons, J.-E. Wahlund, A. Eriksson, L. Eliasson, L. Blomberg, P. Kintner, and H. Höfner, Observations of an upward-directed electron beam with the perpendicular temperature of the cold ionosphere, Geophys. Res., Lett., 22, 2103-2106, 1995. 
Burch, J. L., P. H. Reiff, and M. Sugiura, Upward electron beams measured by DE-1: a primary source of dayside region-1 Birkeland currents, Geophys. Res. Lett., 10, 753, 1983.

Carlson, C. W., J. P. McFadden, R. E. Ergun, M. Temerin, W. Peria, F. S. Mozer, D. M. Klumpar, E. G. Shelley, W. K. Peterson, E. Möbius, R. Elphic, R. Strangeway, C. Cattell, and R. Pfaff, FAST observations in the downward auroral current region: Energetic upgoing electron beams, parallel potential drops, and ion heating, Geophys. Res. Lett., 25, 2017-2020, 1998.

Elphic, R. C., et al., The auroral current circuit and field-aligned currents observed by FAST, Geophys. Res. Lett., 25, 20332036, 1998.

Ergun, R. E., C. W. Carlson, J. P. McFadden, F. S. Mozer, G. T. Delory, W. Peria, C. C. Chaston, M. Temerin, R. Elphic, R. Strangeway, R. Pfaff, C. Cattell, D. M. Klumpar, E. G. Shelley, W. K. Peterson, E. Möbius, and L. Kistler, FAST satellite observations of electric field structures in the auroral zone, Geophys. Res. Lett., 25, 2025-2028, 1998.

Gorney, D. J., Y. T. Chiu, and D. R. Croley, Jr., Trapping of ion conics by downward parallel electric fields, J. Geophys. Res., 90, 4205, 1985.

Hultqvist, B., R. Lundin, K. Stasiewicz, L. Block, P.-A. Lindqvist, G. Gustafsson, H. Koskinen, A. Bahnsen, T. A. Potemra, and L. J. Zanetti, Simultaneous observations of upward moving field-aligned energetic electrons and ions on auroral zone field lines, J. Geophys. Res., 93, 9765-9776, 1988.

Johnstone, A. D., and J. D. Winningham, Satellite observations of suprathermal electron bursts, J. Geophys. Res., 87, 2321-2329, 1982.

Klumpar, D. M., and W. J. Heikkila, Electrons in the ionospheric source cone: Evidence for runaway electrons as carriers of donward Birkeland currents, Geophys. Res. Lett., 9, 873-876, 1982.

Knight, L., Parallel electric fields, Planet. Space Sci., 21, 741, 1973.

Lin, C. S., J. L. Burch, J. D. Winningham, and J. D. Menietti, DE-1 observations of counterstreaming electrons at high altitudes, Geophys. Res., Lett., 9, 925-928, 1982.
Lundin, R., and L. Eliasson, Auroral energization processes, Ann. Geophysicae, 9, 202-223, 1991.

Marklund, G., L. Blomberg, C.-G. Falthammar, and P.-A. Lindqvist, On intense diverging electric fields associated with black aurora, Geophys. Res. Lett., 21, 1859, 1994.

McFadden, J. P., C. W. Carlson, R. E. Ergun, C. C. Chaston, F. S. Mozer, M. Temerin, D. M. Klumpar, E. G. Shelley, W. K. Peterson, E. Möbius, L. Kistler, R. Elphic, R. Strangeway, C. Cattell, and R. Pfaff, Electron modulation and ion cyclotron waves observed by FAST, Geophys. Res. Lett., 25, 2045-2048, 1998.

Parker, E. N., The alternative paradigm for magnetospheric physics, J. Geophys. Res., 101, 10,587, 1996.

Robinson, R. M., R. R. Vondrak, K. Miller, T. Dabbs, and D. Hardy, On calculating ionospheric conductances from the flux and energy of precipitating electrons, J. Geophys. Res., 92, 2565, 1987.

Sharp, R. D., E. G. Shelley, R. G. Johnson, and A. G. Ghielmetti, Counterstreaming electron beams at altitudes of $1 \mathrm{R}_{\mathrm{E}}$ over the auroral zone, J. Geophys. Res., 85, 92-100,1980.

Shelley, E. G., R. D. Sharp, and R. G. Johnson, Satellite observations of an ionospheric acceleration mechanism, Geophys. Res., Lett., 3, 654, 1976.

Temerin, M., and C. W. Carlson, Current-Voltage relationship in the downward auroral current region, Geophys. Res. Lett., 25, 2365-2368, 1998.

J. Bonnell, C. W. Carlson, R. E. Ergun, J. P. McFadden, M. Temerin, Space Sciences Laboratory, University of California, Berkeley, CA 94720 USA

R. C. Elphic, MS D466, Los Alamos National Laboratory, Los Alamos, NM 87545 USA

R. J. Strangeway, Institute of Geophysics and Planetary Physics, University of California, 405 Hilgard, Los Angeles, CA 90024 USA

W. Peria, University of Washington, Geophysics Program, Box 351650, Seattle, WA 98195 USA 